\title{
Data Collection and Medium Access Control Solutions for Underwater Wireless Sensor Networks
}

\author{
Eduardo P. M. Câmara Júnior ${ }^{1}$, \\ Advisor: Luiz F. M. Vieira ${ }^{1}$, \\ Co-advisor: Marcos A. M. Vieira ${ }^{1}$ \\ ${ }^{1}$ Departamento de Ciência da Computação - Universidade Federal de Minas Gerais \\ Av. Antônio Carlos, 6627 - Pampulha - Belo Horizonte - 31270-010 - Brazil \\ Abstract. Underwater wireless sensor networks (UWSNs) can enable many \\ applications in underwater environments. They face many challenges due to \\ the characteristics of these environments and their use of acoustic or optical \\ communications. Here we propose solutions for two existing problems in \\ UWSNs. One of them is CAPTAIN, a cluster-based routing solution for \\ underwater optical-acoustic sensor networks (UOASNs) that explores the best \\ of each communication technology to improve data collection. We also propose \\ UW-SEEDEX, a MAC protocol that employs random time slot schedules to \\ allow nodes to predict each other's transmission schedules to avoid collisions. \\ Through simulations, we show how both proposed solutions perform better than \\ other solutions from the literature.
}

\section{Introduction}

Underwater wireless sensor networks (UWSNs) consist of sets of nodes with sensing, processing, storage, and wireless communication capabilities. These nodes are spread over a region to acquire data and perform other required tasks.

Although wireless communication provides great flexibility for UWSNs, they also introduce many challenges. First, as energy is a very scarce resource, communications must be efficient. Another challenge comes from the direct impact of underwater environments' characteristics like salt concentration, pressure, and temperature in communications [Lanzagorta 2012].

Also, since radio-frequency (RF) systems have some serious disadvantages in underwater environments, UWSNs are usually enabled by acoustic or optical communication [Preisig 2007]. Acoustic systems, used by underwater acoustic sensor networks (UWASNs), provide long-range transmissions but with low throughput and high delays. Optical systems, on the other hand, provide high data rates over short range links and often require line-of-sight positioning. Both technologies can also be combined in hybrid communication systems to get the best of each. UWSNs whose nodes use such a hybrid system are here called underwater optical-acoustic sensor networks (UOASNs).

Due to these challenges, many communication solutions developed for terrestrial wireless sensor networks are not efficient when applied to UWSNs. So, it is necessary to either adapt existing solutions to the context of underwater environments or design new and efficient solutions that consider their constraints.

This work addresses two common network problems in different types of UWSNs. The first problem is data collection in UOASNs, which is defined as the process of routing 
data from sensor nodes to a particular node (or a set of nodes), denominated the sink node. The second problem addressed here is medium access control (MAC) in UWASNs, which consists of defining sets of rules for nodes to efficiently access a shared medium.

The main contributions of this work are:

- CAPTAIN, an algorithm for data collection in UOASNs; its techniques for building clusters, routing establishment, and data collection; and the evaluation of the algorithm through simulations, whose results show the benefits of using CAPTAIN instead of the shortest path algorithm.

- UW-SEEDEX, a MAC protocol for UWASNs that avoids collisions with low overhead by using random time slot schedules; the evaluation of the protocol, via simulations, showing that it can deliver more packets than other protocols from the literature, using, on average, fewer transmissions and consuming less energy.

This work is organized as follows. In $§ 2$, we discuss previous works related to the solutions proposed. $\$ 3$ proposes a cluster-based data collection algorithm, named CAPTAIN, for UOASNs. In $\S 4$, we propose UW-SEEDEX, a MAC protocol for UWASNs. Finally, $\S 5$ we present our final remarks and list possible future works.

\section{Related Work}

Currently, there are many routing protocols that can be used to collect data from underwater acoustic networks [Khan et al. 2018]. Although these protocols can be used in UOASNs, they may suffer from inefficiency since they were not designed to take advantage of the two types of communication provided by these networks. To the best of our knowledge, only a few routing algorithms were proposed for UOASNs. MURAO [Hu and Fei 2012] is a cluster-based routing algorithm that performs data collection in UOASNs. Unlike CAPTAIN, it requires nodes to be spread so that the existence of gateway nodes (nodes in the intersection of two clusters) is guaranteed and considers that only cluster heads are equipped with both types of modems, while cluster members have only acoustic receivers and optical transceivers. [Wang et al. 2017] present another routing algorithm for UOASNs that, different from CAPTAIN, does not use data aggregation since it is focused on the delivery of images and videos. Unlike the other routing algorithms for data collection cited here, CAPTAIN is the only one that combines data aggregation and data collection for UOASNs.

[Jiang 2018] presents a broad study on MAC protocols for underwater acoustic networks. He claims that MAC solutions developed for RF-based wireless networks are not suitable for underwater networks due to the peculiarities of the acoustic channels in the underwater environment.

Code Division Multiple Access (CDMA)-based MAC protocols, such as DPC MAC [Wei et al. 2008], allow collision-free simultaneous transmissions to occur, but need to address issues such as spreading of code assignments and power control of transmissions. Carrier Sense Multiple Access (CSMA)-based MAC solutions, such as CSMA-ALOHA [Azad et al. 2011], can avoid collisions by sensing the channel, but they can cause the hidden and the exposed terminal problems. Reserve-based protocols, such as SF-MAC [Liao and Huang 2012], are effective at eliminating collisions at receivers, but they are affected by network topology as negotiations between the nodes are required for reservation [Jiang 2018]. MAC solutions based on TDMA, such as 
LT-MAC [Mao et al. 2015], can minimize collisions in UWASNs, but they require the use of time synchronization (SYN) services. UW-SEEDEX uses a TDMA scheme to avoid collisions, and, unlike both solutions, it employs a random slot assignment based on a pseudorandom number generator to avoid the need for constant control message exchanges.

During the literature review, we could not find any MAC protocol for UWSNs that uses random slot schedules. However, there are other protocols, such as ARNS [Kosowsky et al. 1988] and SEEDEX [Rozovsky and Kumar 2001], that target other types of networks. ARNS is a MAC protocol designed for satellite networks, while SEEDEX, which served as inspiration for our proposed solution, is a MAC protocol for terrestrial ad hoc networks. As both ARNS and SEEDEX are focused on networks that have different characteristics from the UWASNs, they do not directly deal with factors such as the high propagation delays faced by these networks. UW-SEEDEX, on the other hand, considers the delay propagation and, different from SEEDEX, adds acknowledgments to transmissions in time slots and employs an improved information dissemination scheme to deal with the characteristics of underwater acoustic channels.

\section{CAPTAIN: A Data Collection Algorithm for UOASNs}

CAPTAIN is an algorithm to perform data collection in UOASNs. It is designed for networks with multiple dense groups of nodes (clusters), where optical links can be used for data exchange within groups and acoustic links possibly connect various groups. The algorithm aims to explore the long range of acoustic transmissions and the high bandwidth of optical communication.

CAPTAIN is based on clustering and uses a data aggregation scheme to reduce the overall message traffic and save energy. The algorithm is composed of a configuration period and an operation period. Nodes organize themselves to create routes in the former so they can collect data in the later.

We can consider that CAPTAIN has three phases. In its first phase, it starts the configuration period by dividing the network into clusters, classifying nodes as cluster heads or members. To do so, some node (the sink node, for example) must begin the neighborhood discovery process by broadcasting a discovery message to the nodes around it. When a node A receives a discovery message sent by a node B, it registers B as an acoustic neighbor and uses information about the location of $B$ to check if it is also an optical neighbor (is within the range of its optical modem). After receiving the discovery messages from its neighbors, a node uses the information gathered, together with that on how much energy it has, to calculate its score. This score is sent to the node's neighbors and used to determine which nodes are becoming cluster heads and which ones are becoming cluster members. A node will be a cluster head if none of its optical neighbors has a score higher than its own, or a cluster member otherwise (nodes ID can be used for a tiebreaker).

After forming the clusters, nodes go to the next phase, where they establish routes to deliver data to the sink node(s). They first define the routes within the clusters and then create routes connecting the cluster heads. To create the routes within the clusters, their members use only the information already available to them, thus not requiring a new message exchange. Each member defines its neighbor with the highest score as being the 
next hop for its messages. This definition creates routes to take data from cluster members to cluster heads. To connect the cluster heads, nodes build a routing tree with the sink node as the root. To do this, they shall use acoustic communication to broadcast and receive tree construction messages containing information about the length of the possible paths, in hops, to the sink node and then choose the best of them to join.

After joining the routing tree, a node can move to the third phase. This phase marks the beginning of the operation period, where nodes will start sending data to the sink node. Besides sending the own collected data, some nodes must also forward data collected by other nodes. In the tree routing, each node must forward all the data from its descendants (the nodes belonging to the subtree where it is the root). This requirement creates a potential problem because it can cause the nodes of the highest levels of the tree to have a large volume of data to send and, consequently, much higher energy consumption than the others. To avoid a large number of messages traveling on the network, cluster heads must aggregate the data collected by their clusters before forwarding them to their next hops. Assuming that all nodes perform periodic data collections, the data aggregation helps to reduce the number of transmissions that a cluster head needs to do every round from $n_{c}$ to $m$, where $n_{c}$ is the size of its cluster and $m<n_{c}$. This phase also allows new nodes to enter the network and provide a head rotation scheme to prolong the network lifetime.

\subsection{Simulation and Results}

We implemented and evaluated CAPTAIN using a simulator ${ }^{1}$., comparing its performance with that of a Shortest Path Algorithm (SPA). Results showed that CAPTAIN was able to save more energy in many scenarios, consuming up to approximately $70 \%$ less than SPA in dense networks. This result is very important for underwater sensor networks since their nodes' life are very restricted. We could also observe that CAPTAIN could take more advantage of clusters in the networks than the SPA, resulting in more optical transmissions and less acoustic transmissions. Also, the average latency was generally lower when using CAPTAIN than when using SPA in networks with clusters. CAPTAIN also achieved rates of data collection per hour close to the ideal ones in these networks, using, on average, fewer acoustic transmissions than SPA. Therefore, CAPTAIN is suitable for underwater optical-acoustic sensor networks and has better performance when nodes are deployed in clusters.

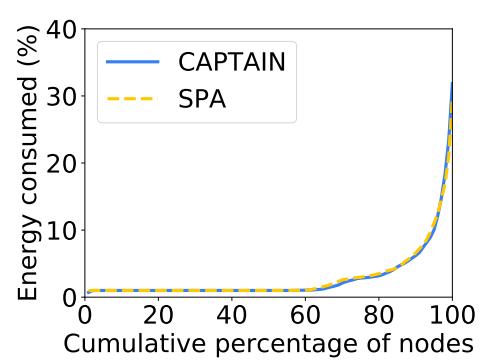

(a) No clusters

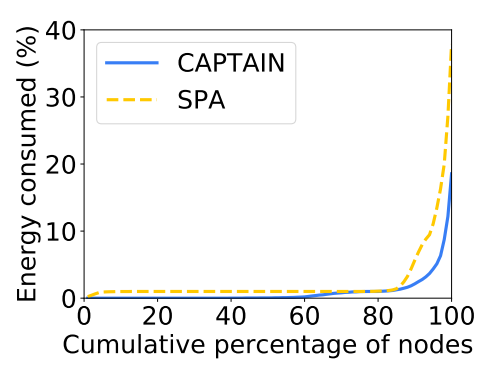

(b) Clusters of 5 nodes

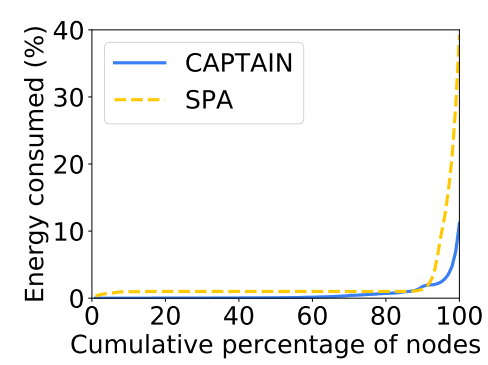

(c) Clusters of 10 nodes

Figure 1. Cumulative percentage of nodes that spent a portion of their energy during the period of network activity.

\footnotetext{
${ }^{1}$ Available at https://github.com/epmcj/captain-sim.
} 


\section{UW-SEEDEX: A Pseudorandom-Based MAC Protocol for UWASNs}

UW-SEEDEX is a TDMA-based MAC protocol designed for UWASNs. Inspired on the existing MAC protocol for terrestrial networks named SEEDEX [Rozovsky and Kumar 2001], UW-SEEDEX avoids collisions with low overhead by employing random time slot schedules produced from seeds. As SEEDEX, the proposed MAC protocol employs random schedules driven by pseudorandom number generators so that nodes can easily publish their schedule just by sharing their seeds. Therefore, nodes can opportunistically decide when to transmit.

The schedules are sequences of slots that define two possible states for nodes: "Listening" $(L)$ or "Possibly Transmitting" $(P T)$. Nodes must remain silent (do not transmit) when in $L$ states, while they may send packets to others in $P T$ states.

The key idea of this scheme is to use random time slot schedules that are created based on pseudorandom number generators. Each node initially chooses a seed and then uses it together with some method to generate its slot schedule. This method can be a Bernoulli process, where each slot state is selected based on a probability parameter $p$ of it to be, for example, a $P T$ one. If all nodes use the same generation method, then they only need to exchange their seeds to determine the entire schedules of others.

After knowing the seeds of all the nodes in its two-hop neighborhood, a node is able to decide the good moments to transmit packets. These moments are the ones where the source (S) and the destination (D) nodes are, respectively, in states $P T$ and $L$. $\mathrm{S}$ can use its seed and the D's seed to predict both slot schedules and thus easily find such slots. S can also check the schedules of D's neighbors to determine how many of them will also be in the $P T$ state and so transmit the packet with probability equal to $p_{t}=\min (\alpha /(n+1), 1)$, where $n$ is the number of other neighbors of the destination also in the $P T$ state, $\alpha$ is a parameter used to control how aggressive a node can be while trying to transmit, and the min function restricts the probability values to valid ones.

To cope with the characteristics of the underwater acoustic channel, UW-SEEDEX adopts a slightly different seed dissemination scheme than SEEDEX and considers the propagation delay and acknowledgments when determining the time slot length and in the process of information dissemination. The UW-SEEDEX operation cycle, shown in Figure 2, is composed of two components that are interspersed over time: an update and a communication interval. Update intervals are periods where nodes exchange information about their seeds and states so they can better plan their future transmissions. They do this by broadcasting information packets to their neighbors while listening to broadcasts from others. Each update interval contains multiple phases where nodes broadcast their known information (seeds and states) once and listen to transmissions from others. The use of multiple phases aims to ensure the adequate dissemination of information since the underwater acoustic channel has characteristics that hinder the correct reception of data.

Communication intervals are sequences of time slots that nodes can use to transmit or receive packets. These slots define $P T$ or $L$ states, just as in SEEDEX. Their length must be at least as long as the time required for sending one data packet and, if confirmation is required, also receiving an acknowledgement (ACK) for it. Therefore, it must take into account the maximum time to transmit a packet, the maximum propagation delay, the maximum time to transmit an ACK, and a guard time to account 


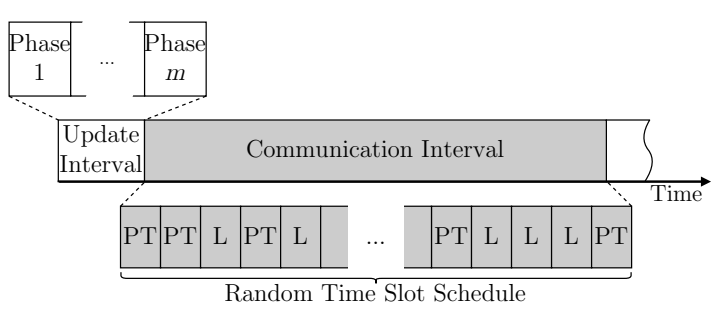

Figure 2. UW-SEEDEX operation cycle.

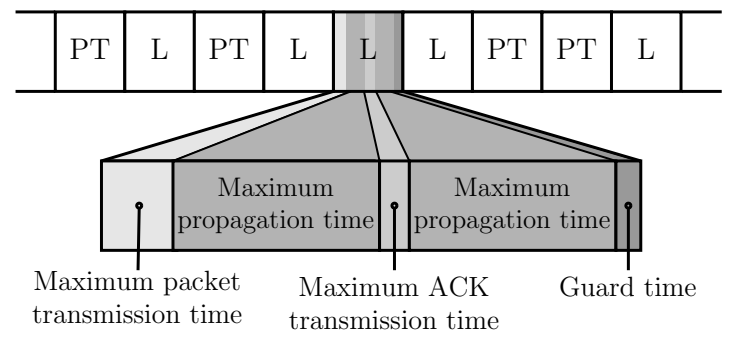

Figure 3. Time slot for one successful transmission.

for possible variations in the propagation delay and any clock drift. Figure 3 illustrates the composition of a time slot where one packet and one ACK can be transmitted. Unlike SEEDEX, UW-SEEDEX considers the time for transmitting ACKs inside the same time slots of data packet transmissions to avoid possible very long waits for confirmations. If ACKs were to be transmitted alone in other slots, the low capacity of underwater acoustic channels, coupled with the random nature of the protocol, could make waiting for confirmation take too long and thus impair applications that need them.

\subsection{Simulation and Results}

We performed simulations using the ns-3.30 simulator to evaluate the performance of UW-SEEDEX in multiple test scenarios. We implemented UW-SEEDEX and two other MAC protocols from the literature: Slotted FAMA (SFAMA) [Molins and Stojanovic 2006] and UW-Aloha [Peng et al. 2009]. More details about the simulation settings can be found in Section 5.3 of the dissertation.

Through extensive simulations, we show that the protocol can deliver more packets than protocols such as within the same time window, using, on average, fewer transmissions than both of them and with low energy consumption. Figure 4 shows the performance of the three MAC protocols in networks with different node densities (using grid deployments with different grid spacings). We also extensively evaluated the protocol parameters via simulations. UW-SEEDEX presented reception rates close to $100 \%$.

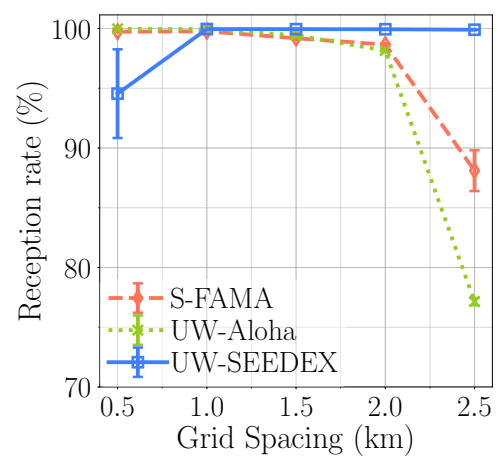

(a)

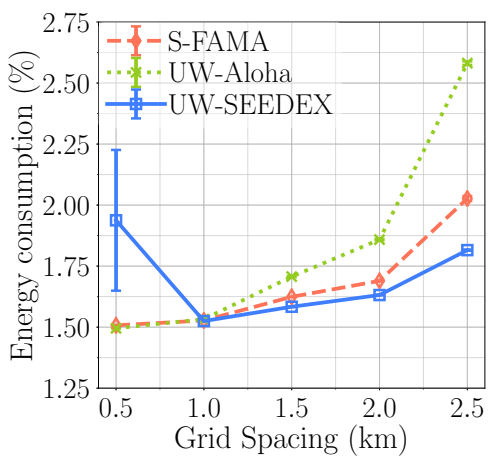

(b)

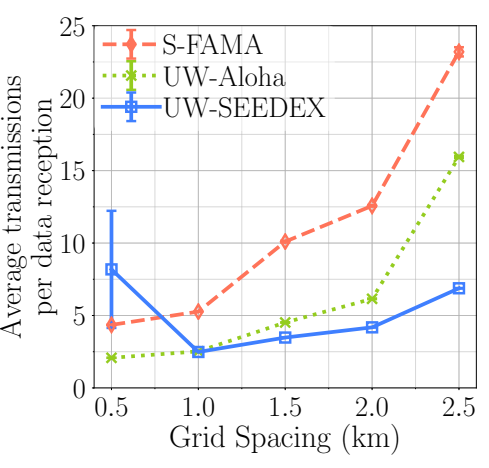

(c)

Figure 4. Performance of the MAC protocols as a function of the network density. 


\section{Conclusions and Future Work}

We first proposed a data collection solution for UOASNs named CAPTAIN. It clusters networks, builds a routing three from the sink node, and then relays the collected data and maintains the routes. We evaluated CAPTAIN by comparing it with the shortest path algorithm (SPA) through simulations. Results show that CAPTAIN can save more energy (principally in dense networks), presents lower average latency, and achieve data collection rates close to the ideal ones using, on average, fewer acoustic transmissions than SPA. Therefore, CAPTAIN is suitable for UOASNs.

Next, we proposed the MAC protocol for UWASNs called UW-SEEDEX. It employs pseudorandom time slot schedules so that nodes can predict other schedules and consequently avoid collisions. We also used simulations to evaluate UW-SEEDEX. Results showed that UW-SEEDEX could deliver more messages than Slotted FAMA and UW-Aloha using fewer transmissions and consuming less energy.

For CAPTAIN, our future research directions include the intention to (i) evaluate the use of different score functions and to (ii) investigate how the solution performance is affected by aspects such as the number of clusters and the average cluster diameter. Moving to UW-SEEDEX, we intend to evaluate (i) the insertion of a new sleeping state in the schedules and (ii) the use of adaptive configuration parameters to adjust transmissions according to network traffic conditions.

\section{Publications}

1. Câmara Júnior, E. P. M., Vieira, L. F. M., and Vieira, M. A. M. (2020). CAPTAIN: A data collection algorithm for underwater optical-acoustic sensor networks. Computer Networks, 171:107145. (Qualis A2)

2. Câmara Júnior, E. P. M., Vieira, L. F. M., and Vieira, M. A. M. (2021). UWSEEDEX: a pseudorandom-based MAC protocol for underwater acoustic networks. IEEE Transactions on Mobile Computing, pages 1-1. (Qualis A1)

3. da Silva Santos, E. R., Câmara Júnior, E. P. M., Vieira, M. A. M., and Vieira, L. F. M. (2019). Aplicações de monitoramento de tráfego utilizando redes programáveis ebpf. XXXVII SBRC. SBC. (Qualis A4). In Portuguese.

4. Câmara Júnior, E. P. M., Vieira, L. F. M., and Vieira, M. A. M. (2019). Topology control in underwater sensor networks using cellular automata. Journal of Cellular Automata, 14. (Qualis B1)

5. Câmara Júnior, E. P. M., Vieira, L. F. M., and Vieira, M. A. M. (2019). 3DVS:Node scheduling in underwater sensor networks using 3D voronoi diagrams. Computer Networks, 159:73 - 83. (Qualis A2)

6. Tavares, R. C., Carvalho, M., Câmara Júnior, E. P. M., de Britto e Silva, E., Vieira, M. A. M., Vieira, L. F. M., and Krishnamachari, B. (2019). FWB: Funneling Wider Bandwidth algorithm for high performance data collection in Wireless Sensor Networks. Computer Communications, 148:136 - 151. (Qualis A2)

7. Vieira, M. A. M., Castanho, M. S., Pacífico, R. D. G., Santos, E. R. S., Júnior,E. P. M. C., and Vieira, L. F. M. (2020). Fast Packet Processing with EBPF and XDP: Concepts, Code, Challenges, and Applications.ACM Comput. Surv.,53(1). (Qualis A1) 


\section{References}

Azad, S., Casari, P., Petrioli, C., Petroccia, R., and Zorzi, M. (2011). On the impact of the environment on MAC and routing in shallow water scenarios. In OCEANS 2011 IEEE - Spain, pages 1-8.

$\mathrm{Hu}$, T. and Fei, Y. (2012). MURAO: A multi-level routing protocol for acousticoptical hybrid underwater wireless sensor networks. In Sensor, Mesh and Ad Hoc Communications and Networks (SECON), 2012 9th Annual IEEE Communications Society Conference on, pages 218-226. IEEE.

Jiang, S. (2018). State-of-the-Art Medium Access Control (MAC) Protocols for Underwater Acoustic Networks: A Survey Based on a MAC Reference Model. IEEE Communications Surveys Tutorials, 20(1):96-131.

Khan, A., Ali, I., Ghani, A., Khan, N., Alsaqer, M., Rahman, A., and Mahmood, H. (2018). Routing protocols for underwater wireless sensor networks: Taxonomy, research challenges, routing strategies and future directions. Sensors, 18(5):1619.

Kosowsky, R. P., Jacobs, I. M., and Gilhousen, K. S. (1988). ARNS: a new link layer protocol. In MILCOM 88, 21 st Century Military Communications - What's Possible?'. Conference record. Military Communications Conference, pages 515-519 vol.2.

Lanzagorta, M. (2012). Underwater communications. Synthesis lectures on communications, 5(2):1-129.

Liao, W. and Huang, C. (2012). SF-MAC: A Spatially Fair MAC Protocol for Underwater Acoustic Sensor Networks. IEEE Sensors Journal, 12(6):1686-1694.

Mao, J., Chen, S., Liu, Y., Yu, J., and Xu, Y. (2015). LT-MAC: A location-based TDMA MAC protocol for small-scale underwater sensor networks. In 2015 IEEE International Conference on Cyber Technology in Automation, Control, and Intelligent Systems (CYBER), pages 1275-1280.

Molins, M. and Stojanovic, M. (2006). Slotted FAMA: a MAC protocol for underwater acoustic networks. In OCEANS 2006 - Asia Pacific, pages 1-7.

Peng, Z., Zhou, Z., Cui, J., and Shi, Z. J. (2009). Aqua-Net: An underwater sensor network architecture: Design, implementation, and initial testing. In OCEANS 2009, pages $1-8$.

Preisig, J. (2007). Acoustic propagation considerations for underwater acoustic communications network development. SIGMOBILE Mob. Comput. Commun. Rev., 11(4):2-10.

Rozovsky, R. and Kumar, P. R. (2001). SEEDEX: A MAC Protocol for Ad Hoc Networks. In MobiHoc '01, pages 67-75, New York, NY, USA. ACM.

Wang, J., Shi, W., Xu, L., Zhou, L., Niu, Q., et al. (2017). Design of optical-acoustic hybrid underwater wireless sensor network. Journal of Network and Computer Applications.

Wei, X., Zhao, L., Li, X., and Zou, C. (2008). A Distributed Power Control Based MAC Protocol for Underwater Acoustic Sensor Networks. In 2008 4th IEEE International Conference on Circuits and Systems for Communications, pages 688-692. 